\title{
Minimization of energy costs for UAV management in a conflict task
}

\author{
Viktor Lapshin ${ }^{1, *}$, Stanislav Ivanov ${ }^{2}$ \\ ${ }^{1}$ Don State Technical University, Rostov-on-Don, Russian Federation \\ ${ }^{2}$ Krasnodar higher military school, Krasnodar, Russian Federation
}

\begin{abstract}
This article considers the method of developing an evader control strategy in the non-linear differential pursuit-evasion game problem. It is assumed that the pursuer resorts to the most probable control strategy in order to capture the evader and that at each moment the evader knows its own and the enemy's physical capabilities. This assumption allows to bring the game problem down to the problem of a unilateral evader control, with the condition of reaching a saddle point not obligatory to be fulfilled. The control is realised in the form of synthesis and additionally ensures that the requirements for bringing the evader to a specified area with terminal optimization of certain state variables are satisfiedt. The solution of this problem will significantly reduce the energy losses for controlling an unmanned vehicle, the possible effect is to save $15-20 \%$ of fuel with a probability of 0.98 , to solve the problem of chasing the enemy.
\end{abstract}

\section{Introduction}

[1-4] presents a method of an optimal evader control strategy in the pursuit-evasion game problem, which does not require attaining the global extremum and complying with the condition of reaching a saddle point. The method was developed based on, first, the assumption that the evader knows its own and the enemy's physical capabilities and, second, on the condition that the enemy resorts to the most probable strategy in order to intercept the evader. The above assumptions made it possible to determine an optimal evader control strategy in the analytical form in the non-linear differential game problem. The final values of some of the evader's state variables were terminally limited, and the total time for solving the game problem $t \in\left[t_{0}, t_{k}\right]$ was assumed to be preset and fixed.

At the same time, it is known that finite values of phase variables are not always possible to reach within a given time period $T=t_{k}-t_{0}=$ const in control problems [5-7]. In this case, having performed an effective evasive manoeuvre, the evader may not have enough resources to deliver the useful load to the specified area. Accordingly, the condition of the fixed time interval $T$ set in [1] may make it principally impossible to reach terminal values in certain subproblems, which substantially reduces the practical value of the approach suggested in [1].

\footnotetext{
* Corresponding author: lapshin1917@ yandex.ru
} 
Besides, additional requirements of terminal optimization may be set for certain state variables of the evader. E.g., for many practical applications, the evader's trajectory is to satisfy the requirements of passing through a given terminal area with optimization of certain functions of state variables at a finite time period. The method presented in [1-4] also does not provide the possibility of terminal optimization of certain evader's state variables.

To this end, below is presented the method of developing an evader control strategy in the nonlinear differential game problem, which factors in an additional condition requiring the evader to be brought to a given area with the optimization of certain state variables for an arbitrary time period $T=t_{k}-t_{0}=$ var. The developed method, as well as that presented in [1], does not require reaching the global extremum, but rather takes an approach which sorts criteria by preference $[1,4,6,8]$. It was again assumed that at every moment the evader knows its own physical capabilities and those of the pursuer, and the optimal control laws are admissible and unique, at least for all time values preceding the moment of the encounter.

The problem statement and the assumptions adopted distinguish the problem from the classical pursuit-evasion conflict problems of the differential game theory [6].

\section{Problem Formulation}

The current position of the evader is determined by phase vector $y(t)$, and the enemy's position - by vector $z(t)\left(y \in R^{n}, z \in R^{m}\right)$. The dynamics of both objects in the phase space is described by the system of nonlinear differential equations [4]:

$$
\begin{aligned}
& \frac{d y}{d t}=f_{y}(y, t)+g_{u}(u, y, z, t), \quad y\left(t_{0}\right)=y_{0} ; \\
& \frac{d z}{d t}=f_{z}(z, t)+g_{v}(v, z, y, t), \quad z\left(t_{0}\right)=z_{0},
\end{aligned}
$$

where $f_{y}, f_{z}, g_{u}, g_{v}$ are known continuous functions of their arguments that can be differentiated enough times; $u, v$ are control functions $\left(u \in R^{r}, v \in R^{p}\right) ; t \in\left[t_{0}, t_{k}\right)$ is an independent variable - time; $y_{0}, z_{0}, t_{0}$ are the initial conditions and the initial time of the evasion game, known to the evader in advance in line with the above assumption; $t_{k}$ is a nonpreset finite instant of time.

Consider the constraints on $n_{1}\left(n_{1}<n\right)$ state variables of the evader's phase vector are described in the form of the equalities $[6,9,10,11,12]$

$$
y_{i}\left(t_{k}\right)=\tilde{y}_{i} \text { or } y_{i}\left(t_{k}\right)-\tilde{y}_{i}=\Phi_{i}\left(y, t_{k}\right)=0, i=\overline{1, n_{1}},
$$

where $\Phi_{i}$ is an $n_{1} \times 1$ dimensional vector function and a requirement of terminal optimization (suppose, maximization) of a certain known scalar function

$$
\max \left\{L_{1}\left(y_{n_{1}+1}, y_{n_{1}+2}, \ldots, y_{n_{1}+n_{2}} ; t_{k}\right)\right\} \text {. }
$$

is imposed on the rest of the $n_{2}\left(n_{1}+n_{2}=n\right)$ state variables.

Then, in accordance with the generally accepted approach [4], we shall consider $t_{k}$ an additional state variable limited by the inequality

$$
t_{k} \leq \tilde{t}_{k}
$$


where $\tilde{t}_{k}$ is the maximum possible finite time determined by the evader's physical capabilities.

The pursuer's goal is to minimize the distance between the players, while the evader's goal is to maximize this distance. Therefore, during the entire time of the game, the vector control functions $u(t)$ and $v(t)$ must simultaneously provide optima (maxima and minima) of a certain given non-negative scalar function $L_{2}\left(y, z ; t_{k}\right)$ characterizing this distance.

Thus, given the restraints on the control intensity $\int_{t_{0}}^{t_{k}} \sum_{i=1}^{r} k_{i} u_{i}{ }^{2}(t) \mathrm{d} t$ and $\int_{t_{0}}^{t_{k}} \sum_{j=1}^{p} k_{j} v_{j}{ }^{2}(t) \mathrm{d} t$, the search of optimal admissible strategies of the control of $\bar{u}(t)$ and $\bar{v}(t)$ in the classical game setting must be carried out based on the condition that a saddle point for the maximin is reached $[4,6-10]$

$$
\begin{gathered}
\max _{u} \min _{v}\{J(y, z, u, v ; t)\}, \\
J(y, z, u, v ; t)=L_{1}\left(y, t_{k}\right)+\int_{t_{0}}^{t_{k}}\left(L_{2}(y, z, t)+\frac{1}{2}\left(v^{\mathrm{T}}(t) K_{1} v(t)-u^{\mathrm{T}}(t) K_{2} u(t)\right)\right) \mathrm{d} t,
\end{gathered}
$$

where $K_{1}, K_{2}$ are symmetric, diagonal positively determined matrices of appropriate dimensions, ${ }^{\mathrm{T}}$ is the transpose symbol, the functional $L_{1}\left(y, t_{k}\right)$ is a terminal component of the Boltz functional and depends on the time of solving the control problem in the final section of the trajectory.

\section{The Method of Problem Solving}

Consider that it is necessary to find a solution only from the point view of one player - the evader. Then, using the known approach $[1,4,6]$, we can bring the game problem (1) - (6) down to the problem of search of a control over the generalized dynamical system $\bar{u}(t)$

$$
\begin{gathered}
\frac{d x}{d t}=f(x, t)+g(x, u, \tilde{v}, t), x\left(t_{0}\right)=x_{0} ; \\
x_{0}=\left|\begin{array}{lll}
y_{0} \quad z_{0}
\end{array}\right|^{\mathrm{T}} ; x=\left|\begin{array}{ll}
y & z
\end{array}\right|^{\mathrm{T}} ; x \in R^{n+m} ; f=\left|\begin{array}{ll}
f_{y} & f_{z}
\end{array}\right|^{\mathrm{T}} ; g=\left|\begin{array}{ll}
g_{u} & g_{v}
\end{array}\right|^{\mathrm{T}},
\end{gathered}
$$

where $\tilde{v}(z, y, t)$ is the "best" function of control of the enemy player based on the feedback principle, with the enemy immediately taking advantage of any non-optimal evader's move. The function $\tilde{v}(z, y, t)$ is constructed in line with the method described in [1].

Then the optimal evader's strategy $\bar{u}(t)$ is realized based on a narrower, compared with (6), condition

$$
\max _{u}\{J[u, \tilde{v}, y, z, t]\}
$$

considering the terminal conditions (3) - (5). In this case the Hamiltonian has the following form:

$$
\begin{aligned}
& H(x(t), u, \tilde{v}, \lambda(t) ; t)=-L_{2}(x(t), t)-0.5 \bar{v}^{\mathrm{T}}(x(t), t) K_{1} \bar{v}(x(t), t)+0.5 u^{\mathrm{T}}(t) K_{2} u(t)+ \\
&+ \lambda^{\mathrm{T}}(t) f(x(t), t)+\lambda^{\mathrm{T}}(t) g(x(t), u, \bar{v} ; t), \\
& \bar{v}(x(t), t)=K_{1}^{-1}\left|\mathbf{0}\left[\frac{\partial g_{v}(x(t), v ; t)}{\partial v}\right]^{\mathrm{T}}\right| \lambda(t),
\end{aligned}
$$




$$
-\min _{u}\{H[x(t), u, \bar{v}, \lambda(t) ; t]\}=-\min _{u}\left\{0.5 u^{\mathrm{T}} K_{2} u+\lambda(t) g(x(t), u, \bar{v} ; t)\right\}=\bar{H}(\bar{x}(t), \lambda(t) ; t),
$$

where the optimal phase trajectory $\bar{x}(t)$ and the vector of conjugate variables $\lambda(t)$ are determined by the adjoint equations of the canonical two-point boundary value problem

$$
\begin{gathered}
\frac{d \bar{x}}{d t}=f(\bar{x}(t), t)-G_{v}(\bar{x}(t), \lambda(t) ; t)^{\mathrm{T}} \lambda(t)-\frac{1}{2}\left[\lambda(t)^{\mathrm{T}}\left(\frac{\partial G_{v}(\bar{x}(t), \lambda(t) ; t)}{\partial \lambda} \hat{\otimes} \lambda(t)\right)\right]^{\mathrm{T}}-\frac{\partial \bar{H}(\bar{x}(t), \lambda(t) ; t)}{\partial \lambda}= \\
=f_{x}(\bar{x}(t), \lambda(t) ; t), \quad \bar{x}\left(t_{0}\right)=x_{0},
\end{gathered}
$$

where $\hat{\otimes}$ is the operation of block matrices multiplication introduced in [9].

$$
\begin{gathered}
G_{v}(\bar{x}(t), \lambda(t) ; t)=\frac{\partial g(\bar{x}(t), \lambda(t) ; t)}{\partial v} K_{1}^{-1}\left[\frac{\partial g(\bar{x}(t), \lambda(t) ; t)}{\partial v}\right]^{\mathrm{T}} \\
\frac{d \lambda}{d t}=\left[\frac{\partial L_{2}(\bar{x}(t), t)}{\partial x}\right]^{\mathrm{T}}-\left[\frac{\partial f(\bar{x}(t), t)}{\partial x}\right]^{\mathrm{T}} \lambda(t)+\frac{1}{2}\left[\lambda(t)^{\mathrm{T}}\left(\frac{\partial G_{v}(\bar{x}(t), t)}{\partial x} \hat{\otimes} \lambda(t)\right]^{\mathrm{T}}+\right. \\
+\frac{\partial \bar{H}(\bar{x}(t), \lambda(t) ; t)}{\partial x}=f_{\lambda}(\bar{x}(t), \lambda(t) ; t), \\
\lambda_{i}\left(t_{k}\right)=\left\{\begin{array}{l}
\gamma_{i}, \quad i=\overline{1, n_{1}}, \\
-\frac{\partial L_{1}\left(\bar{x} ; t_{k}\right)}{\partial x_{i}}, \quad i=\overline{n_{1}+1, n_{1}+n_{2}}, \bar{x}=\bar{x}\left(t_{k}\right), \\
\gamma=-M_{\gamma}^{-1} \bar{\gamma},
\end{array}\right.
\end{gathered}
$$

where $\gamma$ is a constant vector of $n_{1} \times 1$ Lagrangian coefficients for terminal (geometrical) constraint (3).

$$
M_{\gamma}=\int_{t_{0}}^{t_{k}} \Lambda^{\mathrm{T}}(t) \frac{\partial g_{u}(\bar{x}, u ; t)}{\partial u}\left[\frac{\partial g_{u}(\bar{x}, u ; t)}{\partial u}\right]^{\mathrm{T}} \Lambda(t) d t,
$$

where $\Lambda, M_{\gamma}$ are $n \times n_{1}, n_{1} \times n_{1}$ dimensional matrices vector correspondingly.

Optimal value $\Lambda$ taking into account (11) presented in the following form:

$$
\begin{gathered}
\bar{\gamma}=\int_{t_{0}}^{t_{k}} \Lambda^{\mathrm{T}}(t) \frac{\partial g_{u}(\bar{x}(t), u ; t)}{\partial u}\left\{u^{\mathrm{T}}(t) K_{2}+\lambda^{\mathrm{T}}(t) \frac{\partial g_{u}(\bar{x}(t), u ; t)}{\partial u}\right\}^{\mathrm{T}} d t, \quad u=\bar{u}(t), \\
\frac{d \Lambda}{d t}=-\left(\left[\frac{\partial f(\bar{x}(t), u ; t)}{\partial x}\right]^{\mathrm{T}}+\left[\frac{\partial g(\bar{x}(t), u ; t)}{\partial x}\right]^{\mathrm{T}}\right) \Lambda(t), \quad \Lambda\left(t_{k}\right)=\left|\begin{array}{ll}
I & 0
\end{array}\right|^{\mathrm{T}},
\end{gathered}
$$

where $I$ is an $n_{1} \times n_{1}$. identity matrix.

The end time of the game $t_{k}$ is found from the additional scalar equation

$$
\frac{\partial L_{1}\left(\bar{x}(t), t_{k}\right)}{\partial t_{k}}+H\left(\bar{x}(t), \bar{u}, \tilde{v}, \lambda(t) ; t_{k}\right)=0,
$$

where $j=j\left(t_{k}\right)$ for $j=\bar{x}(t), \bar{u}, \tilde{v}, \lambda(t)$. 
The existence of the inverse matrix $M_{\gamma}^{-1}$ is determined by the condition of the evader's controllability.

To solve the two-point boundary value problem (10) - (12) we use the approach suggested in [3] allowing to pass to the one-point problem of integrating ordinary differential equations

$$
Q\left(\bar{x}(t), t_{k}\right)=\left|\gamma-\frac{\partial L_{1}\left(\bar{x}(t) ; t_{k}\right)}{\partial x_{i}}\right|, \quad \bar{x}=\bar{x}\left(t_{k}\right),
$$

where $Q=Q(\tilde{x}(t) ; t)$ - given a non-negative scalar function, which characterizes the distance between the players.

Given (13), (10) can be rewritten as follow:

$$
\begin{aligned}
\frac{d \tilde{x}(t)}{d t}=M_{1} & (\tilde{x}(t), Q, t)\left\{\left[\frac{\partial Q(\tilde{x}(t), t)}{\partial x} M_{1}(\tilde{x}(t), Q ; t)-M_{2}(\tilde{x}(t), Q ; t)\right]^{-1} \times\right. \\
& \left.\times\left[-\frac{\partial Q(\tilde{x} ; t)}{\partial x} f_{x}(\tilde{x}, Q ; t)+f_{\lambda}(\tilde{x}, Q ; t)\right]\right\}+f_{x}(\tilde{x}, Q ; t) .
\end{aligned}
$$

The equations for the sensitivity matrices are characterized by the following formal relations:

$$
\begin{gathered}
\frac{d M_{1}}{d t}(\tilde{x}(t), Q ; t)=\frac{\partial f_{x}(\tilde{x}(t), Q ; t)}{\partial x} M_{1}(\tilde{x}(t), Q ; t)+\frac{\partial f_{x}(\tilde{x}(t), Q ; t)}{\partial \lambda} M_{2}(\tilde{x}(t), Q ; t) \\
M_{1}\left(t_{0}\right)=0 ; \\
\frac{d M_{2}}{d t}(\tilde{x}(t), Q ; t)=\frac{\partial f_{\lambda}(\tilde{x}(t), Q ; t)}{\partial x} M_{1}(\tilde{x}(t), Q ; t)+\frac{\partial f_{\lambda}(\tilde{x}(t), Q ; t)}{\partial \lambda} M_{2}(\tilde{x}(t), Q ; t), \\
M_{2}\left(t_{0}\right)=I_{M},
\end{gathered}
$$

$M_{1}, M_{2}$ are sensitivity matrices; $I_{M}$ is an $(n+m) \times(n+m)$ dimensional identity matrix, which results in receiving a suboptimal strategy of the ally $\tilde{x}(t)$ instead of the optimal strategy $\bar{x}(t)$.

To solve the problem in the form of synthesis, we will further use the known method of constructing adaptive prognostic control [13], which will significantly simplify the synthesized algorithm in comparison with the method presented in [1]. The method used here suits this problem because the time for solving it $T=t_{k}-t_{0}$ is not set in advance. An iterative procedure is constructed to search for a suboptimal solution $\tilde{x}^{j}(t)$ in short time intervals $t \in\left[t_{0}^{j}, t_{k}\right]$ with constant recalculation of the current initial conditions $x\left(t_{0}^{j}\right)=x_{0}^{j}$ (where $j=0,1,2, \ldots$ is the iteration index). At each current interval, stabilization is performed only in respect of the found programme trajectory. The iteration interval - the frequency of recalculation of the predicted trajectory $\tilde{x}^{j}(t)$ for the remaining time interval $t \in\left[t_{0}^{j}, t_{k}\right]-$ is determined by the dynamics of the objects, the power of the flight computer and other factors [11]. As the time interval for solving the problem decreases, the piecewise program control formed this way increasingly tends to control in the form of synthesis [13].

For example, we conducted a numerical simulation of the same practical pursuit-evasion example analysed in [1], where the objects are material points of unit mass moving along the horizontal axis under the influence of the controlling forces $u$ and $v$ : 


$$
\begin{gathered}
\dot{y}_{1}=y_{2}, \dot{y}_{2}=u, \quad \dot{z}_{1}=z_{2}, \dot{z}_{2}=v, \\
t \in\left[t_{0}, t_{k}\right], t_{0}=0, t_{k}=T, \\
y_{1}(0)=-1, \quad y_{2}(0)=0, \quad z_{1}(0)=z_{2}(0)=0 .
\end{gathered}
$$

To examine the attainability of the final conditions with the time fixed $T=t_{k}-t_{0}=$ const, various values were set for terminal pairs of the evader's coordinates

$$
\left(y_{1}, y_{2} ; t_{k}\right)=\left\{\left(y_{1}, y_{2}\right)_{1},\left(y_{1}, y_{2}\right)_{2},\left(y_{1}, y_{2}\right)_{3}, \ldots ; t_{k}\right\}, t_{0}=0, \quad t_{k}=T=\text { const. }
$$

The assumption that, if the solution set forth in [1] is chosen, certain pairs of the end values $\left(y_{1}, y_{2} ; t_{k}\right)$ from the set (18) will be out of reach after the evader $y(t)$ carries out the manoeuvre of "evading" the pursuer $z(t)$, was confirmed on the example of such a simple problem. The condition of fixing the time of solving the problem $T=$ const may lead to its becoming principally impossible to solve.

Further an additional condition of optimizing the evader's velocity in the terminal point was set and the condition of the fixed time interval was eliminated:

$$
\max _{u}\left\{L_{1}\left(y_{2} ; t_{k}\right)=y_{2}\left(t_{k}\right)\right\}, \quad t \in\left[t_{0}, t_{k}\right), \quad t_{0}=0, \quad t_{k}=T=\operatorname{var} ; \quad y_{1}\left(t_{k}\right)=y_{1 k} .
$$

Computer simulation of a practical example was carried out in a mathematical package Mathcad 15. In this case, the mutual movement of two opposing objects in the lateral plane was simulated.

Model realizations of evasion of the player-ally from the opponent are presented in figures 1, 2 (linear speeds of movement of objects and coordinates respectively).

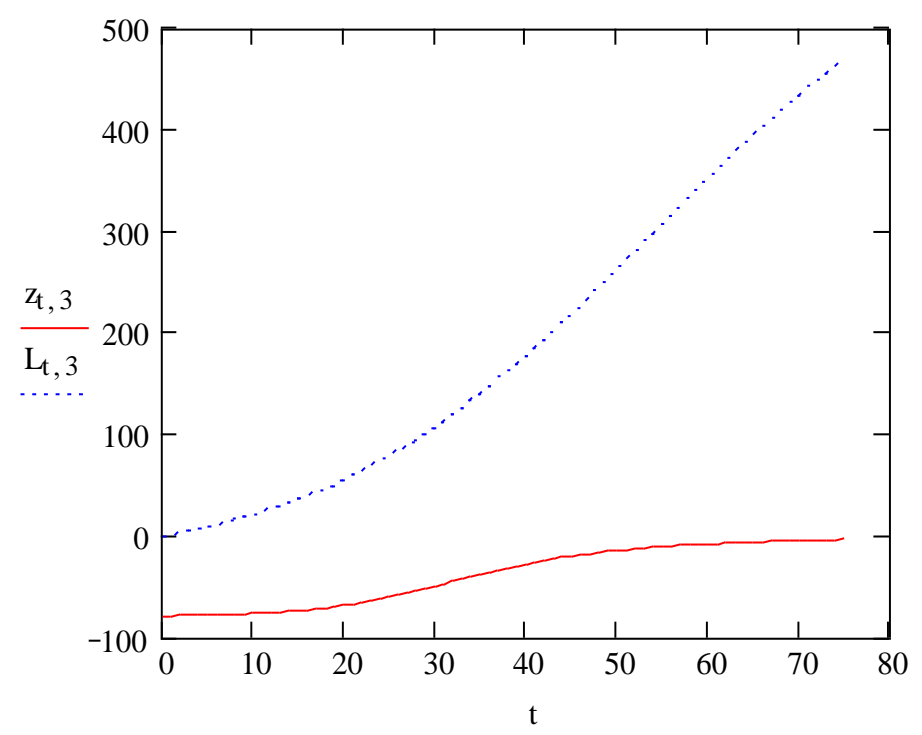

Fig. 1. Linear velocities of objects 


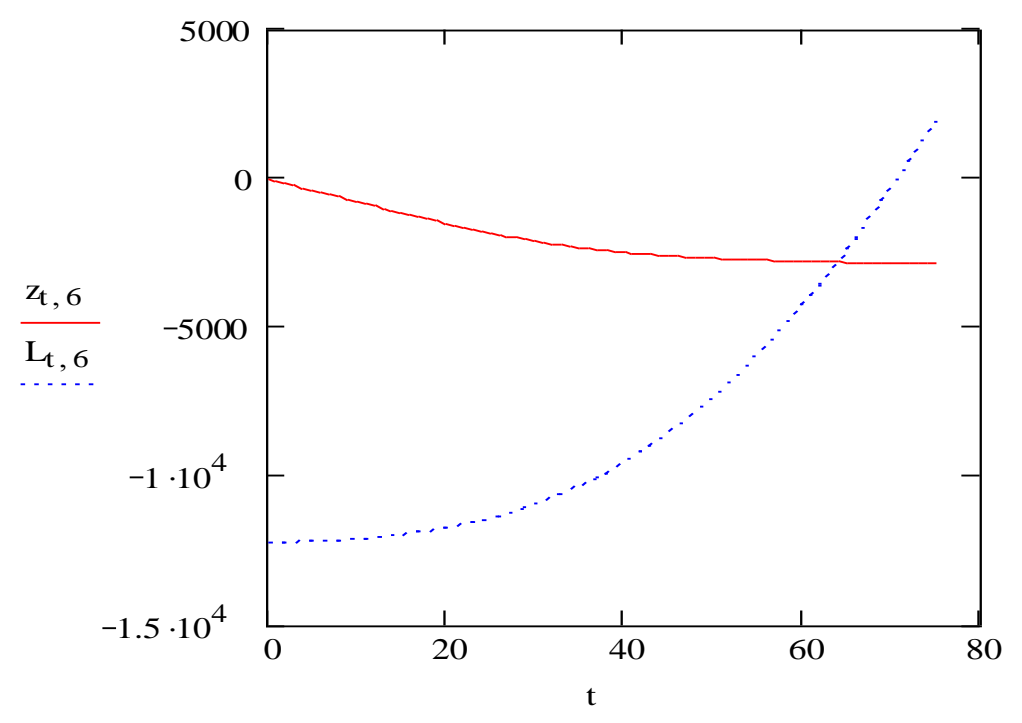

Fig. 2. The Coordinates of moving objects

The errors of bringing into the given terminal point of space and the errors of providing the given parameters of the movement of the ally player were $45 \%-50 \%$ less compared to the errors of the traditional method.

The solution was realized in line with the method described above. It was found that the evader carries out the manoeuvre of "fleeing" from the enemy, then executes the operation of coming to any coordinate $y_{1 k}$ from the set (4.2) with the maximum possible velocity $y_{2}$.

\section{Conclusion}

The solution to the non-linear differential pursuit-evasion game problem was found based on the assumption of the most probable actions of the enemy in order to intercept the evader and taking into consideration the both objects' limited energy for manoeuvring. The terminal optimization of the evader's $n_{2}$ state variables is ensured in the finite region of the phase space, which, in turn, is determined by the constraints in the form of equalities imposed on the remaining $n_{1}\left(n_{1}+n_{2}=n\right)$ variables. The results received in the simulation allow to conclude that the developed method is efficient.

The issues of limited availability of energy resources for bringing the evader to the terminal area were not considered. The solution of this problem will significantly reduce the energy losses for controlling an unmanned vehicle, the possible effect is to save $15-20 \%$ of fuel with a probability of 0.98 , to solve the problem of chasing the enemy.

\section{References}

1. Sokolov S.V., Shcherban I.V. Automatic Control and Computer Sciences, 36, 4. 20-28 (2002)

2. Lapshin V. P. et al. MATEC Web of Conferences. -EDP Sciences, 226, 02012 (2018) 
3. Lapshin V. P., Turkin I. A., Khristoforova V. V. Synthesis of Electromechanical Position Control System by Means of Maximum Principle. Proceedings of the International Russian Automation Conference (RusAutoCon). - IEEE, pp. 1-4. (2018)

4. Shcherban I.V. Journal of Computer and Systems Sciences International, 46, 1. 3-8 (2007)

5. Barkov, V.V. and Kochetkov Yu.A. Theory and control systems, 6. 90-95 (1995)

6. Krasovsky, N.N. and Subbotin, A.I. Positional Differential Games (1974)

7. Panteleev A. V., Bartkowski A. S. Control Theory in examples and tasks (2003).

8. Ananievski I. M. Journal of Computer and Systems Sciences International 4. 19-27 (2000)

9. Baranov V. A. Theory and control systems, 3. 43-59 (2000)

10. Baranov V. A. Theory and control systems, 4. 28-38 (2000)

11. Kuchkarov A. Sh., Rishiev B. V. Automation and remote control, 8. 41-45 (2001)

12. Baratova, E. D., Tarakanov, A. F. Theory and control systems, 5. 30-36 (2003)

13. Bukov, V.N. Adaptive Predicting Flight Control Systems (1987) 